\title{
Vertical cracks characterization and resolution from lock-in vibrothermography
}

\author{
by A. Castelo*, A. Mendioroz*, R. Celorrio**, and A. Salazar* \\ * Departamento de Física Aplicada I, Escuela Técnica Superior de Ingeniería, Universidad del País Vasco \\ UPV/EHU, Alameda Urquijo s/n, 48013 Bilbao, Spain, arantza.mendioroz@ehu.es \\ **Departamento de Matemática Aplicada, EINA/IUMA, Universidad de Zaragoza, Campus Río Ebro, Edificio \\ Torres Quevedo, 50018 Zaragoza, Spain, celorrio@unizar.es
}

\begin{abstract}
This work combines lock-in vibrothermography experiments and a stabilized inversion algorithm to characterize vertical cracks. The ability of the method to retrieve square cracks at increasing depths is analyzed by inverting synthetic data with different levels of white noise. Moreover, the minimum distance between square defects in order to be resolved is determined as a function of depth. Inversions of experimental data obtained at low intensity ultrasound excitation in samples containing calibrated defects confirm the predictions of inversions performed with synthetic data.

\section{Introduction}

Vibrothermography is a non-destructive evaluation technique in which the sample is excited by means of ultrasounds. At the defects, mechanical energy transforms into thermal energy that diffuses inside the sample and produces a surface temperature distribution that can be measured by an infrared (IR) camera. In previous works [1] we calculated the temperature distribution corresponding to modulated closed vertical cracks and developed an inversion algorithm to retrieve the shape and location of heat sources from lock-in vibrothermography data [2]. In this work we analyse the accuracy of the reconstructions of square vertical cracks at increasing depths by inverting synthetic data with added white noise. Moreover, we determine the minimum distance between two equal square cracks for them to be resolved as a function of their depth. The predictions of inversions from synthetic data are confirmed by inverting experimental lock-in vibrothermography data obtained in metallic samples containing calibrated defects.
\end{abstract}

\section{Direct and inverse calculations}

We consider two square (side $L_{i}$ ) and homogeneous (power density $Q$ ) heat sources modulated a frequency $f$, with centres at $y$-coordinates $b_{i}$, and submerged distances $d_{i}$ from the sample surface. They are contained in plane $\Pi(x$ $=0$ ) perpendicular to the surface $(z=0)$ and cover a total area $\Omega$, in a semiinfinite sample of thermal conductivity $K$ and diffusivity $D$. The surface temperature distribution can be written as [1]:

$$
T(x, y, 0)=\iint_{\Omega} \frac{Q}{4 \pi K} \frac{e^{-q \sqrt{\left(\vec{r}-\vec{r}_{0}\right)^{2}}}}{\sqrt{\left(\vec{r}-\vec{r}_{0}\right)^{2}}} d S_{0}=\sum_{i=1}^{2} \int_{b_{i}-L_{i} / 2}^{b_{i}+L_{i} / 2} \int_{-\left(L_{i}+d_{i}\right)}^{-d_{i}} \frac{Q}{4 \pi K} \frac{e^{-q \sqrt{x^{2}+\left(y-y_{0}\right)^{2}+z_{0}{ }^{2}}}}{\sqrt{x^{2}+\left(y-y_{0}\right)^{2}+z_{0}^{2}}} d y_{0} d z_{0}
$$

We solve the ill-posed inverse problem introducing a position dependent heat source distribution, $Q_{f_{k}}\left(\vec{r}_{0}\right)=\Omega\left(\vec{r}_{0}\right) I_{f_{k}}$, and perform the integration in Eq. (1) in the whole $\Pi$ plane. $\Omega\left(\vec{r}_{0}\right)$ is a characteristic function whose value is 1 within area $\Omega$ and zero elsewhere. We tackle the inversion as a minimization problem. Given its ill-posed character, the inversion is stabilized with Total Variation regularization.

\section{Inversion of synthetic data}

Using Eq. (1) we have generated synthetic data corresponding to single, centered square heat sources buried at different depths with $5 \%$ added white noise. Figure 1 shows the normalized plot of $\Omega$ reconstructions, of squares of side $L$ (red contour). Accurate reconstructions are obtained for depths down to $2 L$.

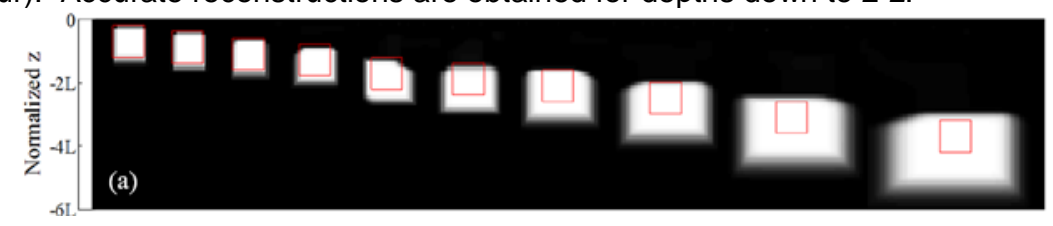

Fig. 1. Reconstructions obtained from synthetic data with $5 \%$ added white noise for a square heat source buried at different depths. Real squares are represented by a red contour line. White corresponds to $\Omega=1$ and black, $\Omega=0$. 
In order to analyse the capability of the method to retrieve separate reconstructions of two equally buried squares we have inverted synthetic data with $5 \%$ added white noise corresponding to two squares buried $0.2,0.6,1.2$, 1.6, 2, 2.6, 3.2mm, and different separation between centers. In figures $2 \mathrm{a}$ and $2 \mathrm{~b}$ we show such reconstructions for $\mathrm{a}$ depth on $1.6 \mathrm{~mm}$ and center separations of 2.8 and $3.4 \mathrm{~mm}$. We set the resolution limit when the value of $\Omega$ between reconstructed maxima is 0.3 . Accordingly, squares in figure $2 \mathrm{a}(2 \mathrm{~b})$ are not resolved (are resolved). Figure $2 c$ represents minimum distances between centers (black lines) and squares configurations (white contours) for two squares to be resolved. Dark gray (light gray) areas represent the locations for resolvable (non-resolvable) defects with $5 \%$ noise.
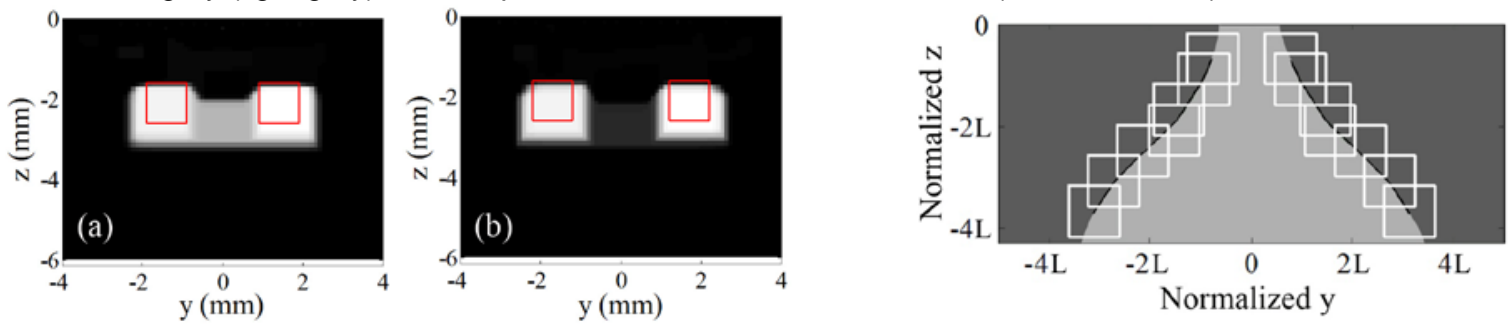

Fig. 2. Synthetic reconstructions (5\% noise) of two squares, $1 \mathrm{~mm}$ side, buried $1.6 \mathrm{~mm}$ beneath the surface with centers separated by $2.8 \mathrm{~mm}$ (a) and $3.4 \mathrm{~mm}$ (b). (c) Limiting distances between squares of size $L$ for being resolved.

\section{Experiments}

The predictions of figures 1 and 2 have been checked by inverting data obtained on metallic samples containing calibrated heat sources. The sample consists of two AISI 304 stainless steel parts with a flat common surface where one or two thin $(38 \mu \mathrm{m}) \mathrm{Cu}$ slabs of known dimensions are placed. The parts are joined by screws. When ultrasounds are launched, friction occurs between the steel parts and the $\mathrm{Cu}$ films. We use a UTvis equipment from Edevis and a JADE J550M IR camera from Cedip. To prevent sample from being damaged, we use low excitation power $(<40 \mathrm{~W})$ and analyse a high number of images in order to improve the signal to noise ratio. In figure 3 we show inversions from experimental data with the Cu slabs located as indicated in red contours (depth of $0.2 \mathrm{~mm}$ in upper and $1.6 \mathrm{~mm}$ in lower figures). Figures $3 \mathrm{a}$ and $3 \mathrm{c}$ correspond to non resolvable distances between centers $(1.15 \mathrm{~mm}$ and $2.5 \mathrm{~mm})$ and $3 \mathrm{~b}$ and $3 \mathrm{~d}$ to distances ( $2 \mathrm{~mm}$ and $5 \mathrm{~mm}$, respectively) where resolution is expected.
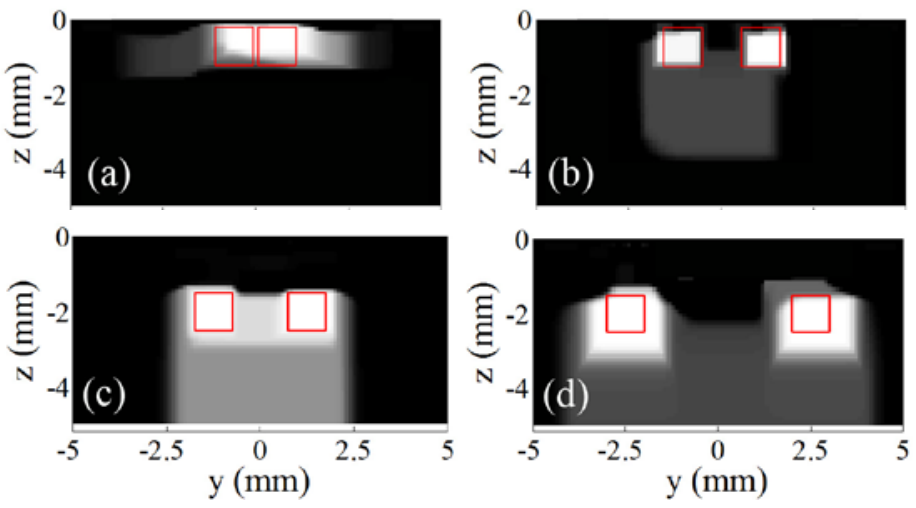

Fig. 3. Real contours and retrieved $\Omega$ corresponding two square $1 \mathrm{~mm}$ side Cu slabs, both buried $0.2 \mathrm{~mm}$ (a and b) and $1.6 \mathrm{~mm}$ (c and d). Center separations of $1.15 \mathrm{~mm}$ (a), $2 \mathrm{~mm}$ (b), $2.5 \mathrm{~mm}$ (c) and $5 \mathrm{~mm} \mathrm{(d).}$

The results in figure 3 confirm the predictions about spatial resolution and are very promising regarding the characterization of vertical cracks from lock-in vibrothermography data.

This work has been supported by the Ministerio de Ciencia e Innovación (MAT2011-23811 and MTM201016917), by Gobierno Vasco (IT619-13 and S-PE11UN024), and by Diputación General de Aragón.

\section{REFERENCES}

[1] Mendioroz A., Castelo A., Celorrio R., Salazar A., "Characterization of vertical buried defects using lock-in vibrothermography. I. Direct problem". Meas. Sci. Tech., vol. 24, pp. 065601-1-11, 2013.

[2] Celorrio R., Mendioroz A., Salazar A., "Characterization of vertical buried defects using lock-in vibrothermography. II. Inverse problem". Meas. Sci. Tech., vol. 24, pp. 065602-1-9, 2013. 